\title{
Characterization of microhollow cathode discharges
}

\author{
M.P. Gomes, B.N. Sismanoglu and J. Amorim \\ Departamento de Física, Instituto Tecnológico de Aeronáutica, \\ Comando-Geral de Tecnologia Aeroespacial, 12228-900, São José dos Campos, SP, Brasil.
}

(Received on 22 April, 2008)

\begin{abstract}
This work is devoted to the study of the electrical characteristics of microhollow cathode discharges (MHCD) at moderate to high pressure in argon and air for different geometries. High-pressure glow discharges can be operated in MHCD devices with $\mu m$ electrode spacing. Experiments have been performed to determine the so-called Paschen curves, i.e. the dependence of the breakdown voltage on the product electrode gap and gas pressure. Current-voltage characteristic curves were obtained as a function of the pressure and hole diameter. MHCD enables stable direct current discharges that could be ignited for pressures ranging from 12 to 800 Torr, in a very wide range of current densities and electrodes materials. Evidence of electron field emission was observed for several ranges of gap spacing.
\end{abstract}

Keywords: Microhollow cathode discharge; high-pressure glow discharge; electron field emission.

\section{INTRODUCTION}

Microhollow cathode discharges (MHCD) devices have attracted attention of researchers due to its possibility to operate at atmospheric and sub-atmospheric pressures [1]. These structures consist of a metallic cathode with a hole in the center and an arbitrarily shaped metallic anode, separated by an insulator (mica in our case). Although often described as MHCD they operate quite differently of conventional hollow cathode discharges. They have three distinct modes of operation: abnormal (at low current), self-pulsed and normal. The transition from abnormal to normal glow discharge is due mainly to hollow geometry that propitiates ions and metastables generation [2]. These particles feed the discharge and in certain experimental conditions may favor the appearance of discharge outside the cathode aperture. The range of discharge operating pressure varies inversely with the hole diameter $D$ of an MHCD, according to Allis-White similarity law. For atmospheric pressure operation the hole diameter must be in order of $100 \mu \mathrm{m}$ [3].

Such microdischarges are in the category of non-thermal plasma, $T_{\text {electrons }}>>T_{\text {gas }}$, and its low cost operation, allied to small size and low power consumption, is an attractive to the plasma applications possibilities in industry, like surface treatment, generation of UV and VUV radiation, reduction of pollutants, gas lasers, biological decontamination, thin film deposition, mainly in a high pressure operation [1, 2]. Microplasmas can be generated in a high-pressure, in rare and molecular gases or in air, choosing cathode hole with reduced dimensions, compared with those of low-pressure operation. In argon gas operation, for example, for a hole with size of about $200 \mu \mathrm{m}$, the discharge may operate at pressure of about 1 bar [3].

This article is organized as follow: in section 2 we present the apparatus used to generate MHCD at low and high pressure and for measurements of electron field emission in the prebreakdown phase. Section 3 makes efforts with interpretation of current versus voltage measurements of MHCD. Arrays of microdischarges were also studied for closed MHCD. Voltage breakdown measurements were performed for different electrode materials and hole diameters characterizing each Paschen's curve. Ionization efficiency for low $p d$ values was compared with conventional plane anode-cathode discharges. Paschen's curves were plotted for electrode spacing of $5 \mu \mathrm{m}$ to investigate a new regime of higher ionization efficiency. Fowler-Nordheim curve was plotted to explain this phenomenon, which is due to electrons field emission.

\section{EXPERIMENTAL SET-UP}

The electrodes of our open MHCD in general are made of approximately $100 \mu \mathrm{m}$ thick molybdenum or cuprum foils separated by a $250 \mu \mathrm{m}$ spacer of mica with holes varying from 200 to $1000 \mu m$ (diameter) through cathode, dielectric, and anode. A closed MHCD in a cathode plane geometry (blind cathode hole for anode hole diameters smaller than 700 $\mu \mathrm{m})$ was also used in these experiments. Sustaining glow discharge voltages were in the range of $200 \mathrm{~V}-400 \mathrm{~V}$ for Ar. Discharge currents $I_{d}$ varied between $0.1 \mathrm{~mA}$ and $8.0 \mathrm{~mA}$. The experiment were performed for an $(p D)$ interval between 0.24 and 56 Torr $\mathrm{cm}$. The operating gas was moderate to highpressure Ar, between 12 and 800 Torr. For field emission experiment $5 \mu \mathrm{m}$ electrode spacing was employed in air. The electrode system was placed in a cylindrical quartz chamber with $10 \mathrm{~cm}$ diameter and $16 \mathrm{~cm}$ length. Before each experiment the chamber (Fig. 1) was cleaned, dried and evacuated to about 1 mTorr. After that, the chamber was filled with pure argon gas and placed at the desired pressure. Resistors of 47

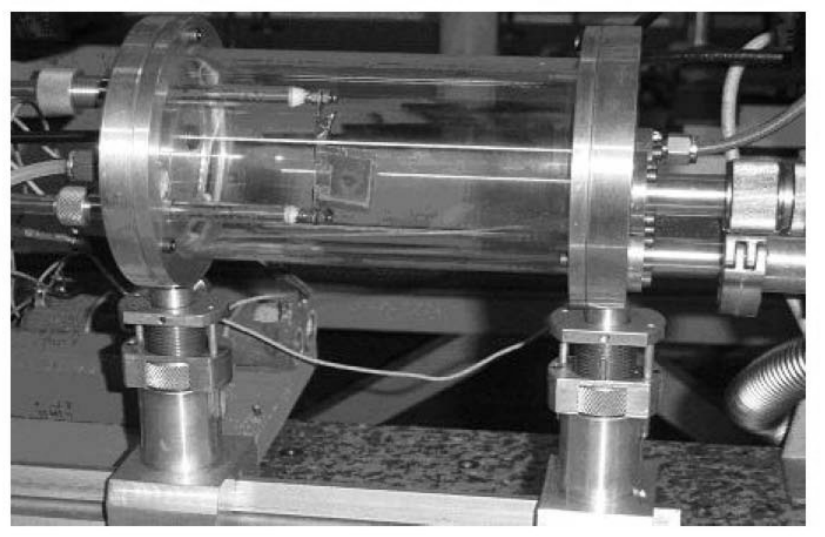

FIG. 1: MHCD device attached to electrodes and seated inside cylindrical quartz discharge chamber. 
$K \Omega$ are used to limit the discharge current.

A digital camera was used to record pictures of the glow discharges. A picoammeter model 414S from Keithley and a digital voltmeter from Minipa were used to measure the current and voltage in the prebreakdown phase. The dc power supply HP 6920B was employed in the experiments of field emission. SEM images, analysis and top view of cathode hole opening was obtained by a JEOL equipment JSM-5310.

\section{RESULTS}

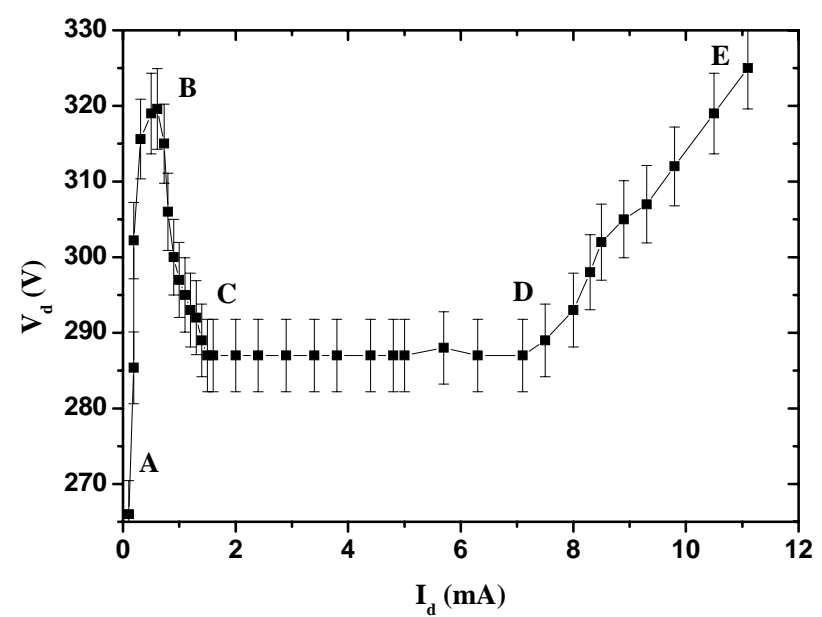

FIG. 2: $V-I$ characteristic curve for a hole diameter of $400 \mu m$ at 40 Torr. For $A-B, B-C, C-D$ and $D-E$ regions, see text.

In Fig. 2 is shown the evolution of the current with voltage applied to an open MHCD in Ar, operated at 40 Torr. As can be seen low current abnormal mode ( $\mathrm{AB}$ region) is present, which is characterized by a positive differential resistance. A self-pulsed mode (BC region) appearing for currents of 0.6 $m A$ can be remarked by a voltage drop with current increase, i.e. negative differential resistance. This region is characterized by high efficiency in producing of ions and metastables species inside the cathode cavity. The confinement of the discharge inside the hole can be seen by the optical appearance of the glow. Increasing the discharge current, one can observe a gradually expansion beyond the cavity, covering the cathode surface. The normal glow discharge mode is seen in the CD region where the voltage is held constant as the current increases. After point D the discharge shifts to a high-current abnormal glow discharge (DE region), where a positive slope in the current-voltage characteristics is observed. We, generally, avoid the abnormal mode of operation, beyond $10 \mathrm{~mA}$ due to overheating of the sample. On the other hand, limiting the cathode surface area, the positive slope in $I-V$ curve is always present.

Fig. 3 shows $I-V$ characteristics of an open MHCD (Mo foils) in Ar in the pressure range of 13-690 Torr. As pressure increases, the sustaining voltage decreases due to more favorable conditions for ionization. They show the typical shape of the abnormal mode, self-pulsed mode and normal mode. The discharge mode characterized by a negative dif- ferential resistance, region $\mathrm{BC}$, was attributed by Schoenbach et al. [1,3] to the onset of the classical hollow cathode effect, where high ionization efficiency is due to pendulum electrons. Meanwhile, calculations showed that cavity electrons do not have enough energy to do ionizing collisions [4]. Aubert et al. [5] showed that $\mathrm{BC}$ region is characterized by self-pulsed mode of operation, where the frequency is driven by the capacitance of the MHCD and by the averaged current delivered by the power supply. Conventional hollow cathode discharges are expected to follow Allis-White similarity law [6], $V=V(p D)$, with $V$ being the sustaining voltage for the discharge. The lowest value of $p D$ is given by the condition that the mean free path for ionization must not exceed the hole diameter $D$. The upper limit for $p D$ is determined by the condition that the distance between opposite cathodes must not exceed the lengths of the two cathode fall regions [7]. High-pressure MHCD operation is expected to be governed by other processes of ionization due to more efficiency in producing ions, photons and metastable excited atoms, causing secondary electron emission from de cathode [6]. In Fig. 3 we note that $p D$ varies between 3 to 7 Torr $\mathrm{cm}$ for hollow cathode mode operation, the limits being larger than theoretical 1 Torr $\mathrm{cm}$ for Ar, indicating that others mechanisms than that explained above are influencing the discharge maintenance. In Fig. 3A one can remark that self-pulsed mode appears for pressures ranging from 180 to 244 Torr, which correspond to $p D$ values from 4.5 Torr $\mathrm{cm}$ to 6.4 Torr $\mathrm{cm}$. Fig. 3B and $3 \mathrm{C}$ show curves having $p D$ values from 3.0 Torr $\mathrm{cm}$ to 7.0 Torr $\mathrm{cm}$. The total uncertainties (statistical and systematic errors) of the measures of $V_{d}$ were of approximately $3 \%$ for the MHCD of $250 \mu \mathrm{m}$ and $2.5 \%$ for 500 and $1000 \mu \mathrm{m}$. These uncertainties were attributed to discharge voltage instabilities.

A closed MHCD consists of a metal-insulator-metal device with a blind hole in the cathode surface (200 $\mu \mathrm{m}$ cathode cavity deep). In this configuration a positive slope is observed in $I-V$ characteristics at the entire pressure range, which enable the operation of discharges in parallel. This means that the discharge itself plays a role of the resistor having the resistance corresponding to the slope of $I-V$ characteristic. Fig. 4 shows the $I-V$ curve for MHCD operated with one and four holes. As can be seen, the positive slope of $I-V$ curve is present in both cases. In four holes operation the sustaining voltage is reduced for a given current, which is distributed to the array of holes. Here, the plasma serves like a resistor of approximately $15 \mathrm{~K} \Omega$ at a pressure of 30 Torr. In this case the plasma was ignited in a four holes plate in Ar, dc operation. Increasing pressure, keeping sustained voltage constant, a higher current is needed to maintain the glow lightening in all holes. It is possible to limit the cathode area covering it with an insulator [8] in order to limit the area available for secondary emission. This way, in open MHCD configuration, a jumping to abnormal mode is observed after gas ignition. Again, a parallel stable operation is possible without ballasting the individual discharges.

The stability and reproducibility of these discharges enable us to develop arrays of microdischarges, with large plasma area for applications. Thus, it is possible to implement arrays of microholes asymmetrically placed in the same MHCD device, like shown in Fig. 4 (right). Here a number of $200 \mu \mathrm{m}$ holes are displayed to form word "plasma" operated in argon gas at a point corresponding to abnormal mode. 

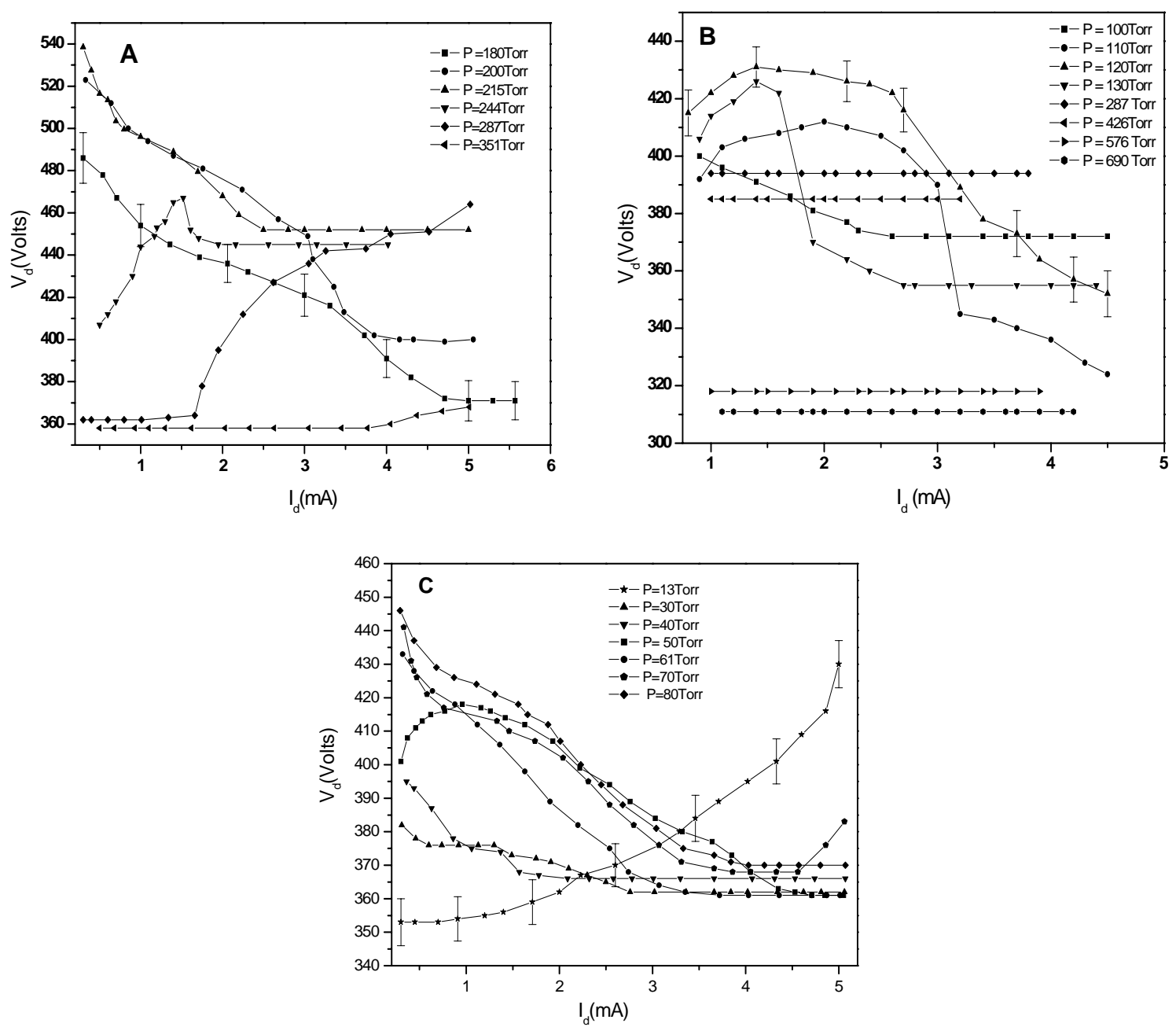

FIG. 3: $I-V$ characteristic curve for open MHCD operated in Ar with hole diameters of $250 \mu m$ (A), $500 \mu m$ (B) and $1000 \mu m$ (C) as a function of current and pressure.

To obtain the Paschen's curves of the MHCD large load resistors were used to limit the current, and hence assuming a Townsend mechanism of breakdown. Fig. 5 shows Paschen's curves for various hole diameters, for MHCD with mica spacer $d=300 \mu m$, in Ar dc operation. The different size holes of MHCD do not change very much the breakdown voltage for $p d$ greater than 0.3 Torrcm. Only the left side aspect of pd Paschen's curves changes when $d$ varies.

Fig. 6 presents Paschen curves for MHCD constructed with different materials. Aluminum (lower work function) is the material that favors the operation at lower voltages while nickel (higher work function) needs high voltages for breakdown. The lower is the cathode material work function the greater is the ionization efficiency. Fig. 7 shows a theoretical Paschen's curve for plane cathode-anode, $d=280 \mu \mathrm{m}$ for air $\left(\gamma=10^{-3}\right)$. The Townsend mechanism commands the breakdown process in these discharges and breakdown voltage $\left(V_{b}\right)$ depends on $p d$, nature of the gas and the electron yield by ion impact $\gamma$. In the same figure a Paschen's curve for a MHCD can be seen. The hole in the center has a diameter $D=200$ $\mu m$. Higher ionization efficiency of the MHCD occurs for low values of $p d$, left-hand branch of the curve, which leads to breakdown voltages lower than plane cathode-anode configurations, while for high $p d$ values the movement of electrons is attenuated due to high pressure and, in this case, the MHCD becomes gradually comparable to a conventional glow discharge, exhibiting the same behavior for breakdown voltages as a function of $p d$.

Fig. 7 also shows a curve for MHCD with electrode spacing of $5 \mu \mathrm{m}$ where lower breakdown voltages is observed for $p d$ values below 1.0 Torr $\mathrm{cm}$ with respect to the configuration with larger gap, $d=280 \mu m$ and plane cathode-anode configuration. Mechanically drilled holes has non-uniform opening surface, with incorporated micro-protrusions on it (Fig. 8). This, allied with the cathode surface roughness, and for a small electrode spacing (of several microns) between electrodes (mica thickness), induces phenomena of electron field emission and tunneling [4]. For this gap, the microscopic electric field becomes very large and the field emission may be responsible for the breakdown in low-pressure operations, instead of only Townsend electron avalanche mechanisms. This same behavior was observed by Baars-Hibbe et 

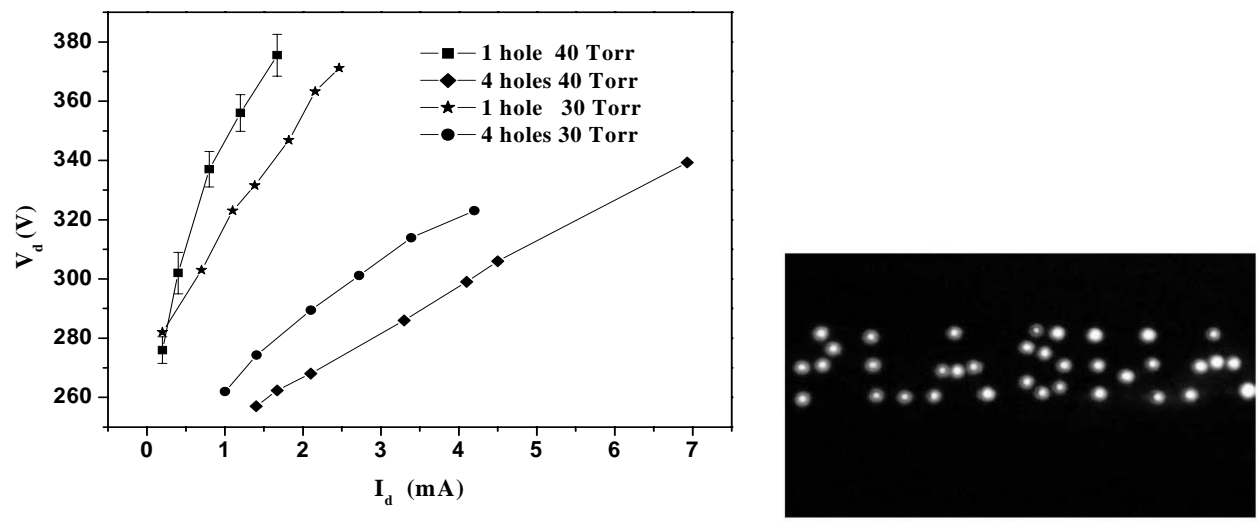

FIG. 4: $V-I$ characteristics for closed MHC dc operated in Ar, with hole diameter of $500 \mu m$ at a pressure of 30 and 40 Torr, for 1 and 4 holes. Closed microhollow cathode, $\mathrm{Cu}$ anode $100 \mu \mathrm{m}$ thick, operated in Ar. Hole diameter $D=500 \mu m$. Mica spacer $250 \mu \mathrm{m}$ thick and cathode cavity deep of $200 \mu \mathrm{m}$. In the right a digital photography shows an association of MHCDs $(D=200 \mu \mathrm{m})$.

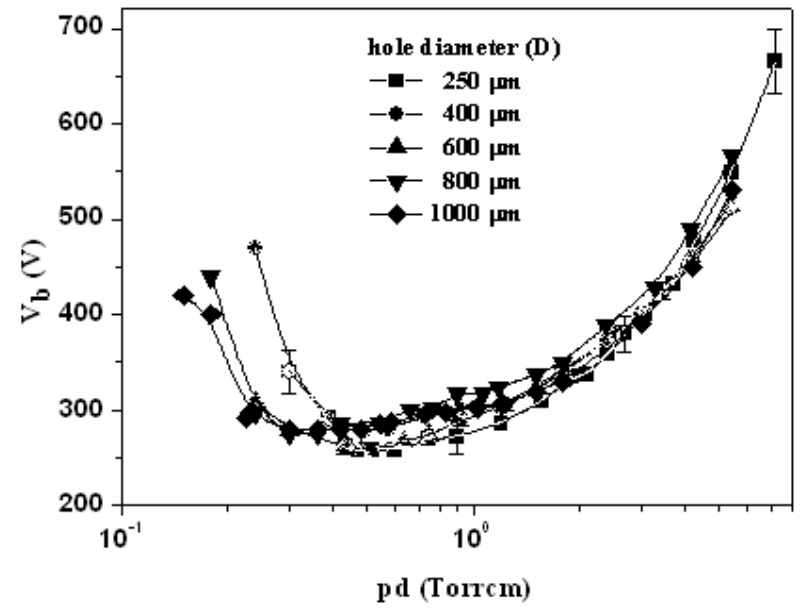

FIG. 5: Paschen's curves for open MHCD operated in Ar for various hole diameters with Mo foil and mica spacer of $300 \mu \mathrm{m}$ thick.

al. [9] in the breakdown of micro structured electrode arrays in the breakdown curves of $\mathrm{Ar}, \mathrm{He}$ and $\mathrm{Ne}$ and by Torres and Dhariwal [10] in microactuators. In accordance to V-I characteristic curve the minimum pressure is about 12 Torr for a MHCD with $D=200 \mu m$.

One can use the Fowler-Nordheim equation (approximately, because this equation holds only for vacuum $[11,12]$ ) and obtain a microscopic electric field just above the cathode surface, responsible for the quantum phenomenon of field emission. In these experiments the total current is given by $I_{d}=\left(\lambda a S F^{2} / \Phi\right) \exp \left(-\mu b \Phi^{3 / 2} / F\right)$, were $a$ and $b$ are universal constants given by $a=1.5414 .10^{-6} \mathrm{~A} \mathrm{eV} V^{-2}$ and $b=6.8309 \mathrm{eV}^{-3 / 2} V n m-1$ [13], $\lambda$ and $\mu$ are generalized correction factors, $\Phi$ is the work function for the relevant point of the emitting surface, $F=\beta E$ is the microscopic electron field, for a field amplification factor $\beta$. The $I-V$ curve was plotted for MHCD in air operated at 20 Torr, $\mathrm{Cu}$ cathode with a single $200 \mu \mathrm{m}$ diameter hole and a very thin electrode spacing $(5 \mu \mathrm{m})$ between electrodes. In Fig. 9 a straight line, with

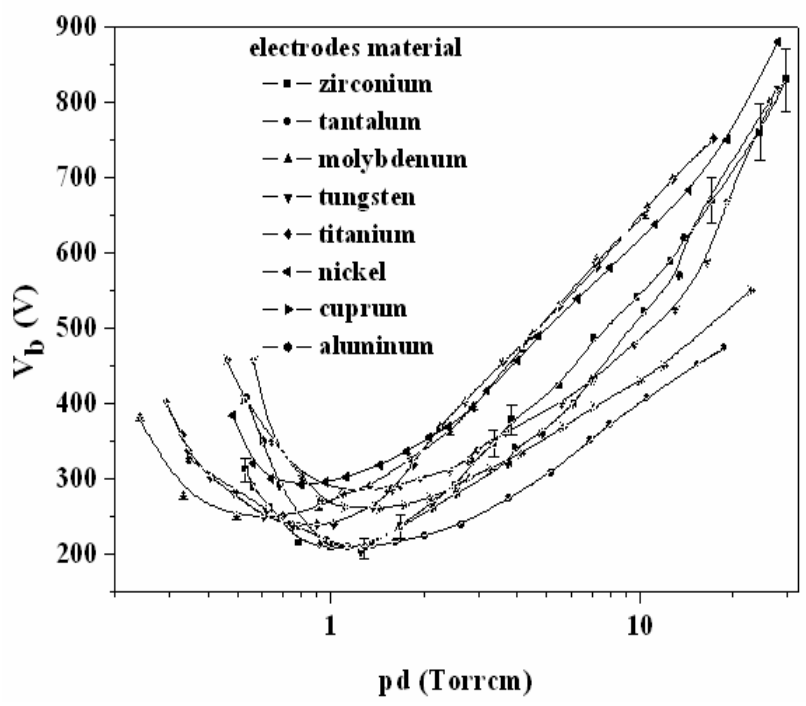

FIG. 6: Paschen's curves for MHCD for different cathode materials. Electrodes: $100 \mu m$ thick; dielectric: $d=250 \mu m$; hole diameter $D=$ $500 \mu m$; gas: Argon.

negative slope, was obtained when $\ln \left(I_{d} / V_{d}^{2}\right)$ is plotted versus $V_{d}^{-1}$, a so-called FN plot. For this device configuration, and using $\Phi=4.6 \mathrm{eV}$ for $\mathrm{Cu}$ electrodes, we obtain $\beta=80$ and $F=10 \mathrm{~V} / \mathrm{nm}$, which is a good value for a local microscopic field needed to start the field emission.

\section{SUMMARY AND CONCLUSIONS}

The $I-V$ characteristic of the open MHCD sustained discharge (Fig. 2) is similar to the well-known characteristic of a low-pressure glow discharge and shows different modes of operation. At low current it presents an abnormal mode followed by a transition to a self-pulsed glow discharge. The abnormal mode is characterized by small current (typically less than $0.5 \mathrm{~mA}$ ). The charge density in the hole is low and does 


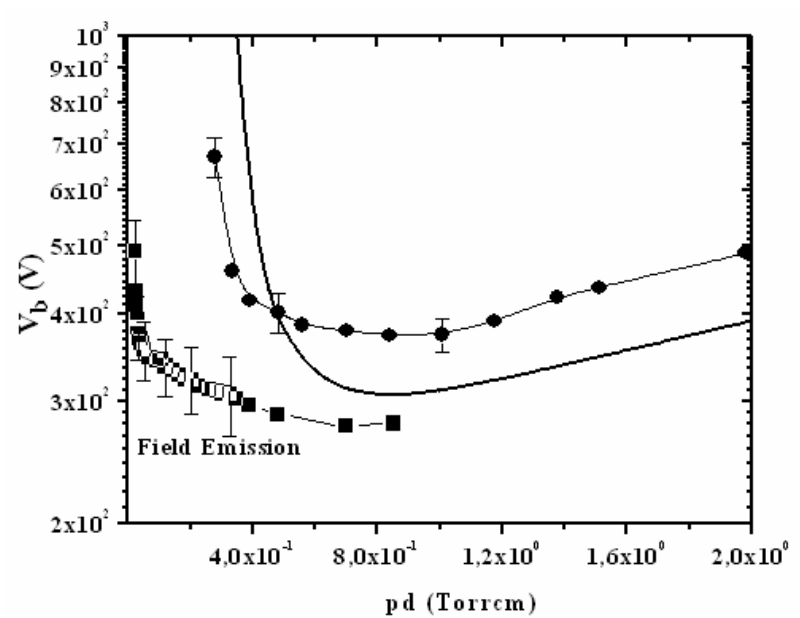

FIG. 7: Paschen's curves for $\mathrm{Cu}$ electrodes in air. Plane theoretical cathode-anode (straight line) and microhollow cathode with $D=$ $200 m(\bullet, \mathbf{\square})$. Full circle electrode spacing of $280 \mu m$, full square electrode spacing of $5 \mu \mathrm{m}$.
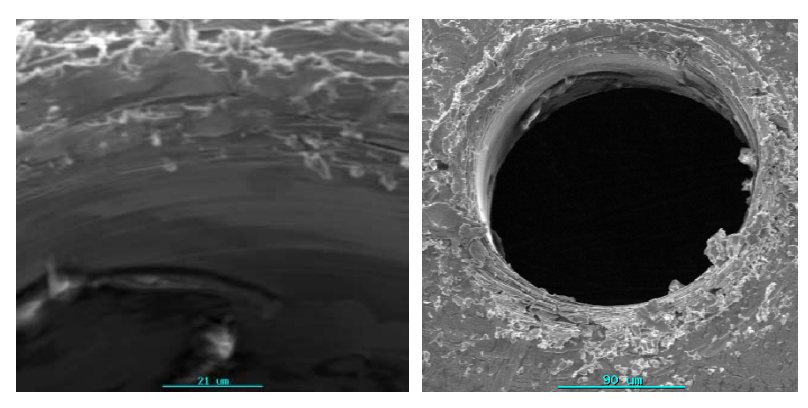

FIG. 8: Scanning Electron Microscopy micrographs of cathode surface showing roughness and protrusions around hole opening.

not disturb the electric field between the electrodes, which is in this case mainly axial oriented. Increasing the current, the axial electric field begins to be distorted by the space charge accumulated inside the cathode and a strong radial electric field develops [14]. The potential distribution in the gap becomes strongly non-uniform and the cathode fall is generated. The enhanced electric field at the cathode facilitates ionization processes and correspondingly a fall in the sustaining voltage and a rise in the current are observed [3].
The breakdown voltage depends on the nature of the gas and $p d$ product. Paschen's curves are given in Fig. 6 for open MHCD dc operated. All of that have the well-known Paschen curve "U" shape. It can be seen that in the region of the minimum, for $\mathrm{Cu}$ and Mo electrodes in Ar gas operation, the breakdown voltage and the $(p d)_{\min }$ product are in reasonable agreement to the ones found in literature [2]. However, in microhollow cathode operations, as in low pressure hollow cathode operations, the left-hand Paschen's curves is shifted to a lower breakdown voltage, compared to a parallel-plate glow discharge [14]. Due to micro protusions existing around the hole opening, inducing an enhacement of the local electric field, the breakdown voltage is reduced. This high-local electric field induces the emission of electrons by tunneling

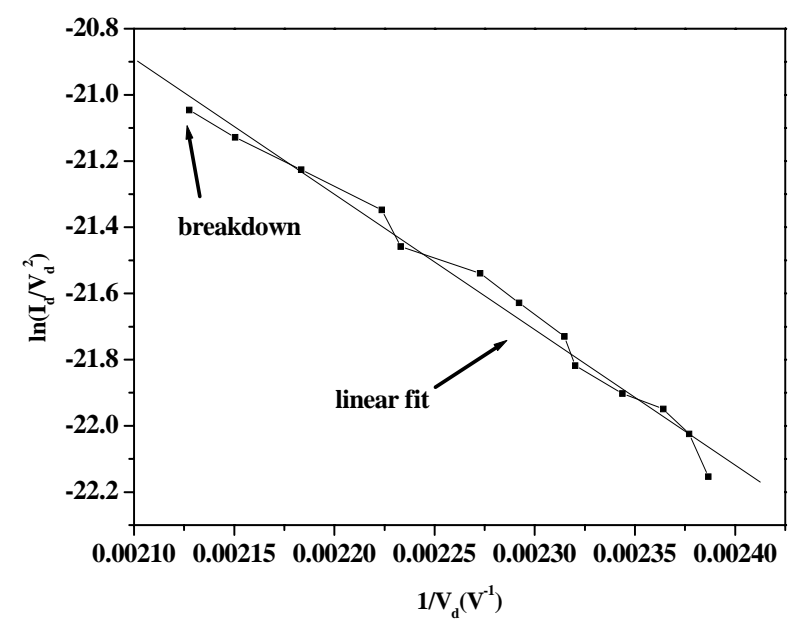

FIG. 9: Fowler-Nordheim plot for $5 \mu \mathrm{m}$ gap device near breakdown.

or field emission process, and for small gaps, be the responsible for the breakdown process. In a future work we will incorporate carbon nanotubes into MHCD hole for enhancing electron emission and reducing breakdown voltages. Spectroscopic measurements of plasma emission spectral lines are in progress in our laboratory to measure discharge parameters in MHCD at high pressure, such as electron density and gas temperature [15].

Authors would like to thank João Paulo Barros Machado of "Laboratório Associado de Sensores e Materiais - INPE", for technical assistance in Scanning Electron Microscopy and helpful discussions.
[1] K. H. Becker, K. H. Schoenbach and J. G. Eden, J. Phys. D: Appl. Phys. 39, R55-R70 (2006).

[2] J. P. Boeuf, L. C. Pitchford and K. H. Schoenbach, Applied Physics Letters 86, 071501 (2005).

[3] R. H. Stark and K. H. Schoenbach, Appl. Phys. Lett. 74, 25 (1999).

[4] B. N. Sismanoglu and J. Amorim, Eur. Phys. J. Appl. Phys. 41, 165 (2008).

[5] X. Aubert, G. Bauville, J.Guillon, B. Lacour, V. Puech and A. Rousseau, Plasma Sources Sci. Technol. 16, 23 (2007).

[6] D. J. Sturges and H. J. Oskam, J. Appl. Phys. 35, 2887 (1964).
[7] K. H. Schoenbach, M. Moselhy, W. Shu and R. Bentley, J. Vac. Sci. Technol. A 21 (4), 1260 (2003).

[8] K. H. Schoenbach, A El-Habachi, M. M. Moselhy, W .Shi and R. H. Stark, Physics of Plasmas 7, 2186 (2000).

[9] L. Baars-Hibbe, P. Sichler, C. Schrader, C. Gebner, K. H. Gericke and S. Buttgenbach, Surf. Coatings Technol. 174-175, 519 (2003).

[10] J. M. Torres and R. S. Dhariwal, Nanotechnology 10, 102 (1999).

[11] P. G. Slade and E. D. Taylor, IEEE Trans. Comp. Pack. Techn. 25, 390 (2002). 
[12] A. J. Wallash, IEEE Trans. Comp. Pack. Techn. 40 (3), 1751 (2004).

[13] R. G. Forbes, Solid-State Electronics 45, 779 (2001).

[14] R. S Pessoa, B. N. Sismanoglu, J. Amorim, H. S. Maciel and G. Petraconi, Gas Discharges, Fundamentals and Applications, ed. J. Amorim, Transworld Research Network, Kerala, India, ch. 7, 175, (2007).

[15] J. Amorim, A. J. Souza Corra, C. Oliveira, B. N. Sismanoglu and M. P. Gomes, Bull. A. Phys. Soc. 38 (2007). 